\title{
Coherent control of nanoscale light localization: creating and positioning isolated sub-wavelength energy hot-spots
}

\author{
Tsung Sheng Kao ${ }^{1, *}$, Stewart D. Jenkins², Janne Ruostekoski ${ }^{2}$ and Nikolay I. Zheludev ${ }^{1}$ \\ ${ }^{1}$ Optoelectronics Research Centre and Centre for Photonic Metamaterials, University of Southampton, SO17 1BJ, U.K. \\ ${ }^{2}$ School of Mathematics and Centre for Photonic Metamaterials, University of Southampton, SO17 1BJ, U.K.
}

We suggest a new paradigm for achieving prescribed localization of optical energy with nanoscale accuracy. Well isolated energy hot-spots as small as $\lambda 10$ can be created and positioned at the chosen meta-molecules on the metamaterial landscape.

In 2002, a method was suggested that based on tailoring phase modulation of ultrashort optical pulses in the time domain to achieve the spatial distribution of the excitation energy in complex nano-systems [1]. Here, in our new approach to control the prescribed optical energy localization, does not depend on a transient redistribution of energy between nano-objects and may be implemented with continuous coherent optical sources, making pulse excitation unnecessary. Implementation of the method requires only widely available instrumentation (a spatial light modulator and a continuous laser) that can be retrofitted to a conventional microscope. Moreover, it does not require the nanoscale system to be spatially inhomogeneous (such as a rough surface) and works with periodic, regular planar array of identical plasmonic resonators opening the opportunities for microscopy, lithography and data storage applications which will be discussed below. It is based on tailoring the spatial variation of the phase in the driving far-field to achieve the prescribed coherent control of localization and depends on the strong interactions between individual plasmonic resonators. This idea has a clear mechanical analogy in two coupled identical oscillators (modelling plasmonic resonators) that may be driven to vastly different amplitudes by setting up a phase delay between otherwise identical coherent mechanical forces driving them. We demonstrate this approach in a planar metamaterial, a two-dimensional array of nanoscale subwavelength meta-molecules excited by turns with spatially phase-modulated optical beam. We show that the near-field energy can be concentrated at chosen meta-molecules while a group of neighbouring meta-molecules remain unexcited. Now by simply adjusting the far-field spatial profile of the excitation beam, the nanoscale hot-spot can be moved between the chosen meta-molecules on the metamaterial landscape. We demonstrate that controllable nanoscale localization of optical energy can be achieved at an artificial nanostructured metamaterial surface, an array of altimetrically split-ring plasmonic resonators when such structure is illuminated with a coherent continuous light wave (a laser) those spatial variation of the phase at the metamaterial plane can be tailored.
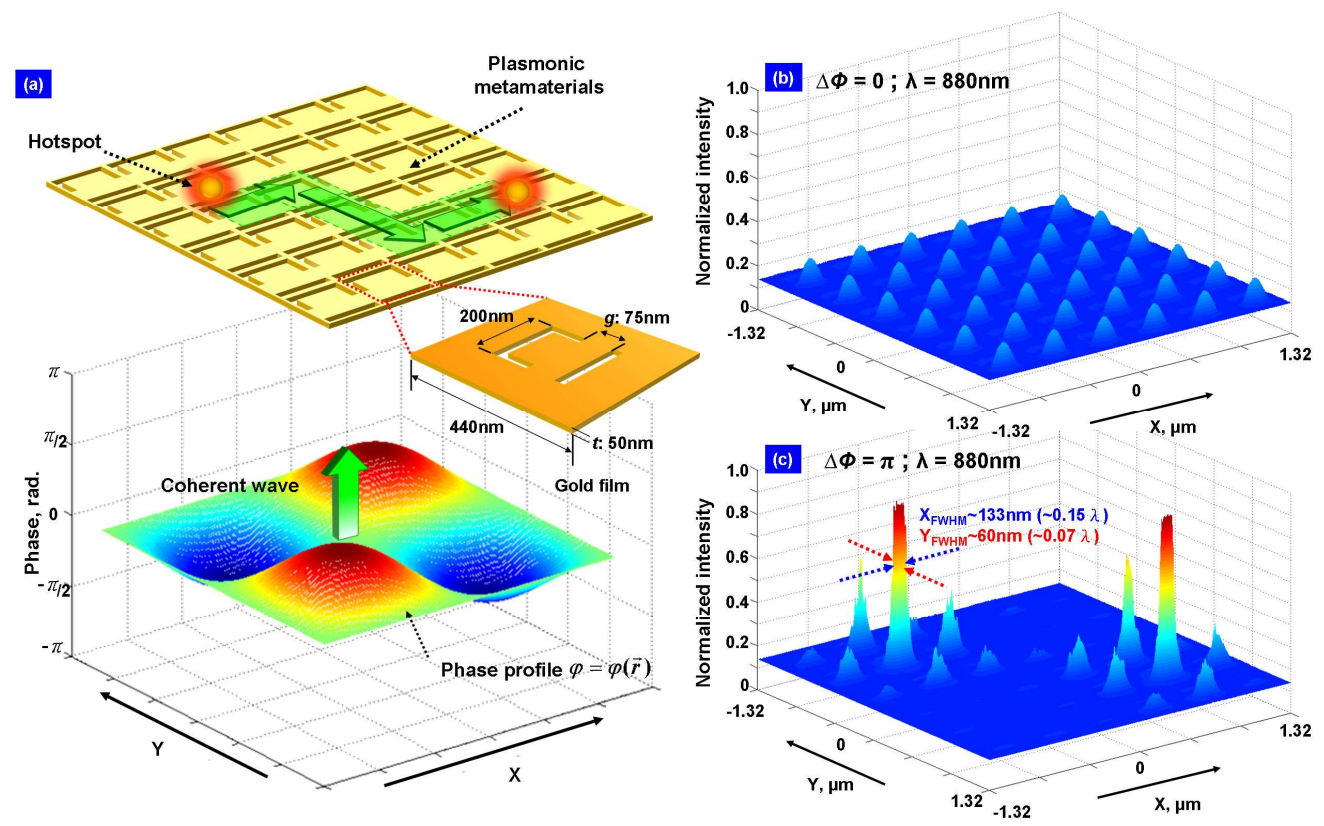

Figure 1. (a) A schematic diagram of the gold split-ring metamaterial and the applied sinusoidal phase profile of the incident wave. The wavelength of incident wave is $880 \mathrm{~nm}$ and the maximum phase difference is denoted as $\Delta \varphi_{\max }$. In (b) the applied phase is flat $\left(\Delta \varphi_{\max }=0\right)$, and the energy distribution is homogeneous across different unit cells. With a large phase difference $\Delta \varphi_{\max }=\pi$, the energy is concentrated in only a few of the unit cells, giving strongly localised peaks (c).

Reference

[1] M. I. Stockman et al., Phys. Rev. Lett. 88, 067402 (2002). 